\title{
HUELLAS NIETZSCHEANAS EN EL PENSAMIENTO DE FOUCAULT
}

\author{
María Lucila Svampa* \\ doi:10.11144/Javeriana.uph32-64.hnpf
}

\begin{abstract}
RESUMEN
La historia y la temporalidad encuentran en el pensamiento de Nietzsche un lugar central, en el que se implican también otras nociones fundamentales para el pensamiento político contemporáneo. En este marco, cobran especial importancia la segunda de las Intempestivas, donde Nietzsche arremete contra el historicismo de su época. Si bien, allí la historia aparece como reguladora de la acción en favor de la vida, esta no se vincularía estrictamente a la memoria, sino al olvido. El objetivo del presente trabajo es recuperar estas nociones a la luz del pensamiento de Foucault. Este muestra en diversos escritos su herencia nietzscheana, tanto en la elaboración del modelo de la guerra, como en la construcción de una genealogía de discursos estratégicos que responden a una voluntad de saber.
\end{abstract}

Palabras clave: historia; acción política; sentido; Nietzsche; Foucault

* Universidad de Buenos Aires - CONICET, Buenos Aires, Argentina.

Correo electrónico: lucilasvampa@hotmail.com

Para citar este artículo: Svampa, M.L. (2015). Huellas nietzscheanas en el pensamiento de Foucault. Universitas Philosophica, 32(64), pp. 235-250, ISSN 0120-5323, ISSN en línea: 2346-2426, doi: 10.11144/Javeriana.uph32-64.hnpf 


\title{
NIETZSCHEAN TRACES IN FOUCAULT'S THOUGHT
}

\author{
María Lucila Svampa
}

\begin{abstract}
History and temporality have in Nietzsche's work an important place, where other fundamental categories are involved as well. His second Untimely Meditation has especial relevance in this framework, where $\mathrm{Ni}$ etzsche warms against the historicism of those years. History in that text is presented as a regulator of action in favor of life, but in spite of that fact, history it is not only associated to memory, but also to forgetting. This paper aims to recover those notions in the light of Foucault's thought. $\mathrm{He}$ shows in different writings his Nietzschean heritage in the elaboration of the war model and the construction of a genealogy of strategic discourses that respond to a will to knowledge.
\end{abstract}

Key words: history; political action; sense; Nietzsche; Foucault 
1. Sobre el sentido y los usos de la historia

La obra de Nietzsche otorga un lugar particular al problema de la temporalidad que, a su vez, alberga otras nociones vitales de su pensamiento. $\mathrm{Si}$ bien, existen numerosos escritos que pueden referirse a este tema, es en la Segunda de las Consideraciones Intempestivas donde Nietzsche ofrece un trabajo pormenorizado sobre la cuestión, generando una fuerte crítica al historicismo de su época. Desde su título mismo, se anuncia la necesidad de una historia en favor de la vida. Mas no implica esto que dicha tarea se circunscriba necesaria y exclusivamente a la memoria: es el olvido que, obedeciendo siempre a una cierta voluntad de poder y conjugado con el recuerdo, puede operar en ese plano. En el presente trabajo se recuperarán tales principios pero, no precisamente limitándonos a la obra de Nietzsche, sino también poniendo en consideración la de Foucault. Tanto en la elaboración del modelo de la guerra, como en la construcción de una genealogía de discursos estratégicos que responden a una voluntad de saber y en la idea de una historia como guía de acción política, el autor de Surveiller et punir pone en evidencia en diversos escritos su herencia nietzscheana. En este marco, la presente contribución apelará a distintos textos a partir de los cuales pueda rastrearse un pliegue entre ambos escritores en lo referente a la caracterización de la historia.

Será acaso oportuno marcar dos puntos de reflexión en lo referente al problema de la historia, que sitúan la afinidad entre estos dos grandes pensadores. En primer lugar, se podría ubicar el estudio sobre el modelo de la guerra para analizar el poder. Este aspecto puede reenviar, en el caso de Foucault, a la idea de guerra de razas, algo que vuelve sobre la noción de vida, también relevante en el pensamiento nietzscheano y que, de hecho, se anuncia ya en el título de la Segunda de las Consideraciones Intempestivas, Von Nutzen und Nachteil der Historie für das Leben (en adelante $V N N$ ). En este mismo punto será propicio dar con algunas precisiones en torno a la historia. Esto, sin obviar la importancia de la genealogía como soporte teórico para el abordaje de dicha problemática, que acompañará la conformación de ciertos discursos que se valen de la historia. En segundo lugar, y como continuación del elemento anterior, algunas precisiones sobre la cuestión del conocimiento, complementarán el apartado precedente. En esta instancia será posible evaluar una lectura pragmática de la historia, no en términos de un imperativo moral ni de un utilitarismo, sino en favor de usos estratégicos ligados a efectos de verdad. Esta verdad será estudiada bajo el cristal que más agudamente permita aproximar el conocimiento a la idea de una invención y no de un origen. 
Veamos cómo se manifiestan en Foucault las evocaciones al pensamiento de Nietzsche. En principio, puede decirse que aparecen variadas, amplias y dispersas menciones al germano en sus publicaciones. Ahora bien, existen algunas presentaciones donde dichas alusiones aparece más in extenso, al conformar este un insumo fundamental para su análisis. Se trata de las conferencias Nietzsche, la généalogie, l'histoire, Nietzsche, Freud, Marx, la primera clase de La vérité et les formes juridiques, y la lección sobre Nietzsche de las recientemente publicadas en español, Leçons sur la volonté de savoir. Tal vez sea oportuno, sin embargo, comenzar con la primera clase de Il faut défendre la société, donde se presenta la hipótesis de Nietzsche. En oposición a otros modos de abordar el poder, la ventaja de esta residiría en que lo entiende como una relación de fuerzas. Las concepciones jurídicas del poder coincidirían en la conformación de un discurso científico que concibe al poder como un bien pasible de ser cedido o transferido, poseído o enajenado. Por otro lado, la perspectiva marxista invocaría en todo tipo de efectos del poder, una funcionalidad económica destinada a mantener cierto tipo de relaciones de producción. Intercambio y economía serían, en suma, los fundamentos del poder en sendos casos.

Una vez marcadas las debilidades de ambas posturas, Foucault presenta otras dos, tras plantear la pregunta ya no sobre qué es el poder, sino sobre el modo en que funciona. Esta vía alternativa desestima entonces la definición precedente (denominada por el par contrato/opresión) y considera una nueva, (caracterizada por el par guerra/represión), que parte de la afirmación de que el poder se define como una relación de fuerzas. Se trata entonces de la hipótesis de Reich y la hipótesis de Nietzsche. Mientras que la primera remite a las producciones que los mecanismos represivos pueden generar, la segunda apunta al poder como combate. En dicho enfrentamiento se expresarían relaciones de fuerzas enfrentadas. Es en este punto donde Foucault apela a la inversión del aforismo de Clausewitz, siendo su resultado una caracterización de la política como la continuación de la guerra por otros medios. De ese esquema se sigue la consideración de que toda situación política lleva inscripta el resultado de una guerra, cuyas cristalizaciones pueden encontrarse en diversas instituciones. Estas precisiones sobre el poder son retomadas en la última clase de este curso, cuando Foucault hace converger, 
por un lado, la guerra como grilla de inteligibilidad para estudiar al poder y, por otro, el poder sobre la vida. Como resultado de esa declinación aparece la idea de una guerra de razas. Habiendo transitado ya las diferencias entre los mecanismos soberanos y disciplinarios, la biopolítica surge como aquella que introduce el elemento de la vida en un sentido netamente biológico. Ahora bien, esta tecnología de poder no hace vivir (y deja morir) sin antes guiarse por ciertas distinciones, que bien podrían ajustarse a los modos de operar del racismo.

Hasta aquí tenemos, pues, dos grandes elementos: la interpretación del poder bajo el esquema de la guerra y su inscripción en la vida. No es difícil en estos puntos afirmar el cuño nietzscheano de dichos presupuestos, que encuentran su asidero en los análisis de la voluntad de poder ${ }^{1}$, por un lado, y en la afirmación de la necesidad de una historia útil para la vida, por el otro. Esta última idea estará asociada a la noción de saber absoluto hegeliana que, junto con el historicismo, conforman un cuadro ante el que Nietzsche va a presentar una fuerte oposición. Esta determinada y "perversa” valoración de la historia está evidentemente acompañada por una idea de verdad, de historia y de hombre muy distinta a la que él sostiene. La parálisis que de acuerdo con Nietzsche ha propiciado el hegelianismo, está basada en un uso de la historia que anestesia la vitalidad crítica con la que debería abordarse. Sus principales reclamos respecto de la historia se inscriben en la necesidad de dar por tierra con la idea de evolución marcada por diferentes etapas que progresivamente alcanzarían un estado final y que, según él, derivarían en una enfermedad de la voluntad. De acuerdo con el planteo presentado en $V N N$, este es el punto de partida para alegar que el problema del sentido en la historia desoye las necesidades vitales de los pueblos, transitando el pasado constantemente y de forma paradójica, como en un estado de parálisis. En el diagnóstico médico que da Nietzsche, las acometidas de esta tradición han dado como resultado una falta de atención en el presente a la hora de estudiar el pasado, de modo tal que se vuelve impensable construir una historia efectiva que potencie fuerzas creativas para el tiempo actual. Con pretensiones metahistóricas, este historiador no hace más que desarraigar al presente de su lazo vital con el pasado y deja así de lado la capacidad formativa de la historia. Este es el com-

1 Estos análisis son trabajados fundamentalmente en Zur Genealogie der Moral, Die fröbliche Wissenschaft (en adelante $F W$ ) y en Der Wille zur Macht. 
portamiento que define al hombre histórico en el que, de forma muy distinta a Hegel, Nietzsche propone pensar: se trata de un hombre que concentrado en el pasado, se encuentra atrapado en la espera de un bienestar siempre por venir. Por el contrario, el hombre suprahistórico no distingue entre pasado y presente y no concentra la mirada en un prometido estadio futuro, sino que "da a la existencia el carácter de lo eterno e idéntico".

El exceso de historia que denuncia Nietzsche como síntoma de decadencia vital es el de la sobresaturación histórica. Hay una impotencia desatada por la tensión interior y exterior que derivan en inconvenientes para la personalidad. El individuo, temeroso y replegado en su mundo interior, cuyos deseos están desligados del exterior, inhibe significativamente sus posibilidades de acción. "La formación histórica de nuestros críticos no permite ya más que se llegue a ningún efecto en sentido propio, es decir, a un efecto sobre la vida y la acción" (Nietzsche, 2003, p. 83). Además, esto provoca la perturbación de los instintos del hombre y una notable dificultad para llegar a la madurez, aspectos que se manifiestan en el impulso destructivo de la época. Solo alejado de la justicia de la moderna historia, el hombre se envuelve en sanas ilusiones y es capaz de entregarse al ímpetu creador. De otra manera, los hombres, enmarcados en los fines del tiempo pierden la posibilidad de la novedad. Nietzsche afirma que la llamada justicia histórica destruye todo lo vivo, lo vuelve antinatural. Este modo de abordar la historia no se guía por la ilusión del amor, sino que presenta un modo de relación entre teología e Historia que enmarca al conocimiento en una religión, arte o genio, algo que deriva en la esterilización de lo vivo, elemento que, sin dudas, plantea el rechazo de Nietzsche con respecto al cristianismo hegeliano ${ }^{2}$.

En este contexto, el abordaje de la historia se vincula sin dudas al de la genealogía. Foucault (2004) resume las diferencias entre ellas del siguiente modo. En primer lugar, comenta que la historia rechaza el punto de vista suprahistórico, absoluto, algo de lo que la genealogía conforma una perspectiva solidaria. Esto implica que no hay una constante por reconocer, que atraviese el destino de la

2 "Y es que todo lo vivo necesita a su alrededor una atmósfera, una aureola llena de misterio. Si se le retira esta envoltura, si se condena a una religión, a un arte, a un genio, a girar como un astro sin atmósfera, no nos deberíamos sorprender si acontece su petrificación y se seca, convirtiéndose en estéril" (Nietzsche, 2003, p. 99). 
humanidad y en la que la historia efectiva se sostenga; por el contrario, lo que ella pretende es partir de fragmentos que piensen a la humanidad desde lo discontinuo. En segundo lugar, la idea de acontecimiento es leída como encadenada a un continuum, como parte de un desarrollo teleológico que lo sobredetermina; la historia efectiva lo denomina en cambio a partir del surgimiento de su singularidad, como fuerzas que se disputan en un campo azaroso. Además, mientras que esta última perspectiva apunta a lo más marginal, a los acontecimientos más próximos, la primera dirige su atención a las más grandes jerarquías, a las más altas estructuras. El único modo de convertir esa historia en un análisis genealógico parece ser que el primero se apodere de los productos del segundo para hacer un uso de ellos. Planteada esta tarea, Foucault muestra cómo Nietzsche en la segunda intempestiva presenta tres modalidades de la historia, que luego metamorfosea: la historia monumental se convierte en parodia, la anticuaria deriva en un uso disociativo de la identidad y, por último, el sacrificio del sujeto de conocimiento toma el lugar de la voluntad de conocimiento de la historia crítica. El uso de la historia aquí muestra un giro rotundo respecto de la tradición: "Se trata de hacer de la historia un uso que la libere para siempre del modelo, a la vez metafísico y antropológico, de la memoria. Se trata de hacer de la historia una contramemoria, $-y$, como consecuencia, desplegar en ella una forma completamente distinta del tiempo-" (Foucault, 2004, p. 63).

Lo anterior se manifiesta con la identificación de tres tipos de formas en que se puede abordar la historia: la monumental, la anticuaria y la crítica, relacionadas respectivamente con tres formas en que la historia puede dársele al hombre: como el que actúa y se esfuerza, como quien venera y conserva, y como quien bajo la necesidad de liberarse, sufre. La primera es aquella que toma del pasado lo más grande, en aras de hacerlo nuevamente posible, pero elevando ciertos hechos a la posición de ejemplo a imitar, de tal modo que los pueblos guiados por esta perspectiva corren el riesgo de caer en una doble trampa: de una parte, muchos otros aspectos del pasado se dejan a un lado en detrimento de este esquema de hechos monumentales que se admiran; de otra, la elevación de una colección de efectos -tal como se presenta para estos hombres, que no son capaces de encontrar modelos de acción sino en el pasado-, puede confundirse con ficción por ser desmedidamente embellecidos. La segunda forma de abordar la historia no goza del mismo impulso de acción que la anteriormente descripta; esta conserva y 
venera el origen, al tiempo que dignifica lo singular, pero perdiendo de vista un campo más amplio. Nietzsche señala aquí el riesgo de que, lo que se ponga en aquél lugar, sea sencillamente por viejo, y entonces lo nuevo quede deliberadamente excluido. Sin frescura vital alguna, la historia anticuaria momifica y olvida la tarea de engendrar vida ${ }^{3}$. Finalmente, en tercer lugar, el abordaje de la historia crítica enjuicia al pasado como un modo de habilitar la posibilidad de librarse de él para generar nuevos comienzos. Ahora bien, puesto que estamos inmersos en una formación histórica dada, no es posible desentenderse completamente del pasado, lo cual genera una tensión. Al respecto, Nietzsche sostiene que en esta instancia entran en lucha una primera y segunda naturaleza: un pasado del que puede desearse proceder y del que, de hecho, se procede.

Acerca de este último punto es importante señalar que Nietzsche propone un uso de la historia que deje de lado las veneraciones y que libere al hombre de la enfermedad histórica. De esta manera, formula una profunda crítica al uso de la historia que se realizaba en su época y aboga por un uso ahistórico; en palabras del filósofo: "no hay que no dejarnos enterrar por los muertos". Ese uso excesivo de la historia socava nuestra capacidad creadora y, además, es el peso del pasado quien marca y condena al presente; para contrarrestarlo y poder crecer, el autor defiende el olvido -como falta de conciencia-, sano y necesario. El contexto en que $V N N$ se publica, representó, sin dudas, un estímulo al que Nietzsche responde en tono polémico. Afirma en este escrito que la cultura alemana estaba afectada por una enfermedad cultural, siendo los discípulos hegelianos sus principales causantes por sostener un conformismo epigonal, dice Nietzsche (2003): "Creo que en este siglo no ha existido ninguna variación o giro peligroso de la formación alemana que no se haya vuelto peligroso a raíz de la influencia, hasta el momento enorme, de esta filosofía, la hegeliana” (p. 110).

Recordemos que en el marco del idealismo absoluto de Hegel, el estudio de la filosofía de la historia ha tenido una enorme influencia en la reflexión sobre el tiempo histórico, fundamentalmente, en los pensadores del llamado progreso. Esto es, una tendencia que protagonizaron fundamentalmente Alemania y Francia en los siglos XVIII y XIX, donde se sostenía una idea teleológica de la histo- 
ria. Podría describirse la composición sobre la antinomia de la historia que dicho debate refleja a través de dos posturas que protagonizan una contradicción en torno al sentido: o bien sostienen, -como lo hace el idealismo alemán- un sentido en la historia, o bien, defienden su antítesis (Bouton, 2005). Esta última posibilidad representa lo que Kant denominaba "abderitismo": un absurdo y constante progreso y retroceso ${ }^{4}$. En este contexto, Nietzsche dedica parte de su obra a la reflexión sobre la historia, formulando críticas explícitas al hegelianismo dominante en su época; sin embargo, lo peculiar de su perspectiva es que allí el problema acerca del sentido en la historia -instalado, como se dijo antes, por el hegelianismo- muta hacia la cuestión de sus usos ${ }^{5}$, los cuales se distinguen por ser legítimos o no legítimos, buenos para la vida o embalsamadores de ella.

\section{Historia o fábula de la verdad}

EN UNA LÍNEA MUY SIMILAR - que se vincula con el problema de la función del pensamiento- Foucault propone distanciarse con una serie de discursos que se conforman a través de saberes históricos por la vía de la genealogía. En este sentido, la conformación de los discursos estratégicos estará ligada a efectos de verdad. Este punto puede pensarse siguiendo la ruta de algunos textos en los que Foucault trabaja explícitamente sobre la relación entre conocimiento y verdad a través de Nietzsche. La primera de las conferencias de La vérité et les formes juridiques recupera las prácticas sociales estudiadas a la luz de los dominios de saber.

4 El denominado fatalismo tiene también sus variantes: o hay un Fatum que gobierna a los individuos o simplemente se trata del azar, concepción bajo la que la alternativa del declinar de la historia gracias a la acción de los hombres aparece representada por Rousseau. En cambio, la primera postura permite pensar en un progreso; sobre esta acepción pueden tener injerencia los hombres (Fitche, Schelling) o, por el contrario, puede plantearse dicho avance como obra de la naturaleza (Kant) o de la Providencia (Schlegel, Herder). Bouton ubica a Hegel dentro de un racionalismo para el que la historia puede concebirse objeto de la filosofía por ser accesible al concepto sin identificar una contradicción necesaria con la visión providencialista, por compartir ambas la idea de un sentido de la historia.

5 Sobre el problema de la temporalidad, vale la pena mencionar que en $V N N$ aparece un concepto que se desarrollará más tarde y con más fuerza en $F W$. Se trata del eterno retorno de lo mismo, que presenta múltiples aristas para su análisis; véase: Deleuze, 2008. En el presente escrito no se busca desconocer la relevancia de dicha noción, sino, más bien, reconocer la necesidad de brindarle un análisis en profundidad. 
Con ese objetivo, en relación, por un lado, con la subjetividad y, por otro, con la idea de verdad, evoca los presupuestos principales que predominaron en el pensamiento occidental. En esta instancia, Foucault aborda el pensamiento cartesiano, ya que este postula la posibilidad de alcanzar representaciones absolutas, que constituyen el insumo fundamental de la verdad. Lo anterior haría eclosión en la figura de un sujeto transparente, que puede conocerse a sí mismo y que, por lo tanto, constituye la historia. Foucault busca reconsiderar dicho esquema dislocando su orden, por lo que ya no se trataría de un sujeto capaz de constituir la historia, sino que, más bien, se constituye en la historia. Este proceso se forjaría a través de diversas prácticas sociales, entre las cuales, las judiciales serían las de mayor relevancia. Hace intervenir allí a Nietzsche, que -según afirma Foucaultinaugura un nuevo horizonte teórico tras la crisis del anterior. Con el objeto de ampliar esta afirmación, recurre a un fragmento inicial de Über Wabrheit und Lüge im außermoralischen Sinne en el que Nietzsche afirma: "En algún apartado rincón del universo, desperdigado de innumerables y centelleantes sistemas solares, hubo una vez un astro en el que animales astutos inventaron el conocer. Fue el minuto más soberbio y más falaz de la Historia Universal, pero, a fin de cuentas, solo un minuto" (Nietzsche, 1990, p. 4). Allí el conocimiento, lejos aparecer vinculado a un origen (Ursprung), se asocia a una invención (Erfindung). En pocas palabras: el conocimiento no obedecería a un origen dado, sino a una creación contingente.

Mediante esta cita, Foucault pone en evidencia cómo Nietzsche, lejos de situar en un espacio solemnidad el tratamiento del tema, lo traslada al plano del azar. Muestra que aquella tradición que busca mediante una indagación que se traslada a los orígenes, el seno de la verdad, fracasa. Y dicha falla sucede porque en esa creación no hay más que circunstancias menores y marginales. Esto quiere decir que las circunstancias bajo las que se forja el conocimiento no responden al orden de lo necesario; por el contrario, la cuestión se termina resolviendo en el seno del azar. Entonces, el conocimiento no representaría más que el resultado de un enfrentamiento entre fuerzas, de modo que aquel esquema que sitúa la verdad en una adecuación al objeto a conocer, o bajo categorías fundamentales y esenciales, resulta poco satisfactorio. No se trataría de un proceso de investigación que acumule y avance mediante resultados que lo aproxime a un momento idílico y relevador de creación. Muy por el contrario, esa instancia estaría habitada por 
enfrentamientos y hostilidad de voluntades que luchan por inscribir su triunfo en la historia. Estas premisas representan una cruzada en contra del neokantismo emergente en esa época, que Foucault también identifica en $F W$, una obra posterior del germano, donde aparece tematizado el violento conflicto entre conocimiento y caos. La celebridad de dicho libro es atribuida, generalmente, al parágrafo en el que se anuncia la muerte de Dios. Esa existencia era sostenida por la tradición filosófica que buscaba en esa figura una garantía que asegure la continuidad entre el conocimiento y el objeto a conocer. Esto impacta sin dudas en la concepción del sujeto que se mencionó algunas líneas antes: la unidad de un sujeto que desea y conoce, se ve ahora en problemas. Este trabajo puede fácilmente complementarse con el de la Clase sobre Nietzsche, en la que el modelo nietzscheano se presenta como una alternativa al aristotélico. Aquí, a través de la introducción de conceptos como los de mentira, ilusión, error y apariencia, se afirma, por un lado, la idea de una verdad no verdadera y, por otro, la de un conocimiento que no tiene un origen, sino una historia. El conocimiento no se vincula con la naturaleza humana, nada hay antes de él, tampoco cuenta con un modelo. Puesto que las cosas no tienen un sentido a descifrar ni existen para ser leídas en algún sentido en particular, el conocimiento se conforma a partir de un espacio complejo habitado por la maldad. Es decir que todo aquello que parecía ser el presupuesto de la verdad, ahora es situado como un efecto de ella. De este modo, Foucault señala que Nietzsche suprime la idea de un conocimiento que tenga vocación de verdad, premisa que sustenta la aparición, nuevamente, del elemento de la vida. Por ello, dado que en un juego de relaciones se suceden y se producen fuerzas transitadas por violencia y por dominación, la verdad se constituiría, contra la ilusión de conocimiento y en favor de su sometimiento y síntesis, en un sistema de errores.

Toda cosmovisión se conformaría, entonces, a partir de quien interpreta, siempre desde un punto de vista parcial, limitado y que no admite una verdad universal. Este modo de abordar el asunto, inaugurado por Nietzsche, se denomina perspectivismo. De esta manera, al buscar los vínculos con el derecho y el poder, Foucault altera los modos de abordar el conocimiento, sostiene que:

Si quisiéramos saber qué cosa es el conocimiento, no hemos de aproximarnos a él desde la forma de vida, de existencia ascética característica del filósofo. Para saber qué es, para conocerlo realmente, para aprehenderlo en su raíz, 
en su fabricación, debemos aproximarnos a él no como filósofos, sino como políticos, debemos comprender cuáles son las relaciones de lucha y de poder. (Foucault, 1995b, p. 28)

Respecto a la función del pensamiento, puede afirmarse que en Nietzsche este elemento también produce una interrupción con el horizonte intelectual de su época. Si en Foucault vimos que se plantea la mirada política, en Nietzsche, la figura será la del arquitecto: "Quien carezca de experiencia superior y más vasta que los demás no podrá saber interpretar el pasado, pues este es siempre un oráculo: solo como arquitectos del futuro y como conocedores del presente podréis comprenderlo (...). Formad una imagen que sirva de modelo al futuro y olvidad esa absurda superstición de ser epígonos" (Nietzsche, 2003, p. 94). De modo que solo aquel que, con un impulso creador, logre concentrarse en la construcción del futuro, tiene derecho a juzgar el pasado. Entonces, no es quien siga las huellas del pasado quien podrá interpretarlo de modo no estéril, sino que esto le corresponderá a un hombre reflexivo y centrado en la poderosa fuerza del presente. Con impulso constructivo, este individuo valora las necesidades vitales y logra así proyectar y edificar los tiempos venideros. El derecho a interpretar el pasado surge de una poderosa potencia creativa del presente que sirva de modelo al futuro.

Una de las consecuencias que implica la sobresaturación histórica es la creencia de toda época en su vejez. Haciendo clara alusión al pensamiento hegeliano, Nietzsche afirma aquí cómo la formación histórica es una canosidad desde la infancia, cuando algunos, creyéndose en una atmosfera crepuscular, ejercen una mirada retrospectiva y de balance sobre el sentido histórico.

$Y$ es que la formación histórica es realmente también una especie de canosidad desde la infancia y los que desde niños llevan tal signo tienen que llegar a la instintiva creencia de la vejez de la humanidad. A la vejez corresponde, efectivamente una ocupación crepuscular, esto es la mirada retrospectiva, el balance completo la conclusión, la búsqueda de consuelo en lo ya sido, el recuerdo...en suma, la formación histórica. (Nietzsche, 2003, p. 105)

En Nietzsche, la généalogie, l'histoire, Foucault busca definir el trabajo genealógico en la obra de Nietzsche. Nuevamente, aquí se problematiza la cuestión del origen: debe prescindir de su la búsqueda aquel que quiera acercarse a la verdad. Es decir que se rechaza el presupuesto que pretende dar con una esencia prima- 
ria, anterior a posibles corrupciones o deformaciones, a partir de la cual se podría acceder al conocimiento; se trata de una configuración de fuerzas no necesarias tiene por resultado una multitud de invenciones. Tras rastrear el uso del término Ursprung en muchas de las obras nietzscheanas, Foucault se extiende en la diferencia entre Herkunft, Entstehung y Erbschaft ${ }^{6}$. Esas interpretaciones generan un repertorio que sucintamente responde a resultados de intercambios que generan dominados y dominantes y que crean distinciones y definiciones en función de los resultados de las tensiones. Esos efectos deliberados que operan con los rituales correspondientes para cada procedimiento tienen forma de valores, siendo este el modo en que surgen ideas como la libertad, el bien y el mal.

Este es el proceso por el cual se establecen una variedad de reglas que obedecen a una dominación. Ajustando la interpretación que las pone en marcha, y en función de su propia funcionalidad, aquellos que resultaron como triunfadores se apropian de ellas:

Si interpretar fuera sacar lentamente a la luz una significación enterrada en el origen, solo la metafísica podría interpretar el devenir de la humanidad. Pero si interpretar es apropiarse de un sistema de reglas que en sí mismo no tiene significación esencial, e imponerle una dirección, plegarlo a una nueva voluntad, hacerlo entrar en otro juego y someterlo a reglas secundarias, entonces el devenir de la humanidad consiste en una serie de interpretaciones. Y la genealogía debe ser su historia: historia de las morales, de los ideales de los conceptos metafísicos, historia del concepto de libertad o de la vida ascética, como emergencia de las interpretaciones diferentes. Se trata de hacerlas aparecer como acontecimientos en el teatro de los métodos. (Foucault, 2004, pp. 41-42) 7

6 El primer término refiere al linaje o grupo social de procedencia; el genealogista que apunte a ello buscará un hilo conductor que sintetice aspectos que pueden aparecer como accidentes disociados, mas sin pretender una unión entre ellos, puesto que su intervención tendría el efecto de disparar fragmentos de algo que parecía unido. Entstenung remite, en cambio, a la idea de emergencia; ahora bien, el momento de surgimiento que ese término señala no es inocuo por producirse en una determinada configuración de fuerzas que previamente lucharon por el triunfo en ese enfrentamiento. Vale la pena aclarar que el término Erbschaft, que remite a la idea de herencia, es tan solo aquí mencionado por Foucault pero no desarrollado como sí lo es el resto de las denominaciones por él reunidas.

7 El problema de la interpretación es retomado en Nietzsche, Freud, Marx, donde Foucault apela a la interpretación de la interpretación, que volviendo sobre sí misma conseguiría una apertura de los 
El pensamiento que marca una cercanía con el fin del mundo y su juicio final, que es una idea cristiano-teológica, deriva en una humanidad paralizada y moribunda. Esta tradición expresa la idea de que nuestro tiempo es el último de los posibles y, por consiguiente, está facultado para ejecutar un juicio final sobre todos los tiempos. La humanidad se ve atrapada en este memento mori, que no permite optar por nuevos rumbos en un contexto de sin sentido de todo acontecer. Aquí se anuncia el imperativo del espíritu del nuevo tiempo que tiene que "volver contra sí mismo su propio aguijón". Estos son los riesgos del cinismo, que justifica la idea de una historia como compendio de la inmortalidad de lo dado. El hombre que defiende la vituperable doctrina de la marcha de la historia, obstruye sus fuerzas vitales y joviales y solo desviándose del camino que indica una vida conforme a esta formación histórica, será posible dar batalla al hastío existencial. De este modo, Foucault alega que la miseria de su tiempo actúa como una pesada carga que lleva desde generaciones precedentes y que a la postre anestesia el sentido crítico, no permitiendo distinguir la utilidad y el

signos. Así, el pensamiento de estos tres pensadores alemanes vendría a inaugurar una nueva derivación de la hermenéutica, por instalar de modo radical un cambio en el seno de la interpretación misma. La infinita intervención a la que la filosofía somete a la filología instaura un movimiento que apunta a destotalizar no solo las construcciones semánticas, sino completos regímenes de verdad que sucesivamente operan restringiendo los imaginarios por procurar su legitimidad y conservación. Mas, en el ejercicio que aquí se propone, la interpretación no tiene por finalidad un objeto original, sino que asume una sucesión perpetua de interpretaciones. Es decir que la interpretación no viene aquí a develar el acceso a lo primario, sino que pone de manifiesto un mecanismo que produce en lo referente a los conceptos, domesticaciones de las que el sujeto no sale ileso. Como bien señala Grüner (1995), en definitiva la interpretación es el sujeto, en la medida en que está constituido por las interpretaciones que ensaya sobre su propia relación simbólica con el mundo.

8 "Consecuencias terribles y destructivas tienen que aparecer cuando una creencia semejante, de repente, a través de un vuelco audaz, se diviniza como el verdadero sentido y fin de todo lo acontecido anteriormente, cuando toda la miseria conocida se eleva a la consumación y cumplimiento de la Historia universal" (Nietzsche, 2003, p. 110). Este presupuesto ha arrastrado a los alemanes a una ceguera por la que justifican todo acontecer como un proceso necesario en la marcha de Dios. Se ofrece entonces, en un gesto de adoración divina, obediencia al "poder de la Historia". El fin y el curso de la marcha de la historia en términos hegelianos son así cuestionados por Nietzsche: "Pero este mismo Dios se ha hecho a sí mismo transparente e inteligible dentro de la serena hegeliana y ya ha ascendido todos los posibles escalones dialécticos de su devenir hasta su autorrevelación: de modo tal que para Hegel el punto máximo y final del proceso universal coincidía con su propia existencia berlinesa" (Nietzsche, 2003, p. 111). 
perjuicio de la historia para la vida, que justamente este libro reivindica como función práctica de la historia9.

\section{Comentarios finales}

PARA TERMINAR, SERÍA OPORTUNO VOLVER sobre la lectura que es posible recuperar, en ambos autores, acerca de la historia, y que se deriva del recorrido que se vino haciendo. En el pensamiento de Nietzsche podría encontrarse la idea kantiana del pensamiento como regulador y como guía de la acción práctica. Esto solo es posible con una consabida distinción en lo referente a la moral, puesto que esta tarea ya no tendrá como fin el imperativo categórico, sino el impulso para la vida. Tal como vimos, en este punto el olvido y el perspectivismo tienen una relevancia peculiar, ya que no se trataría de recuperar toda la vida. Gracias al estímulo de una fuerza plástica, el presente es el que interviene en la conformación del conocimiento, que ya no tiene como objeto una verdad absoluta y, tampoco, a un sujeto soberano como protagonista. En el caso de Foucault vemos que, si bien su trabajo sobre la historia se sostiene en gran medida a través de la obra de Nietzsche, no es $V N N$ el texto que más cita. En su perspectiva tampoco existe un imperativo moral en torno al conocimiento de la historia, puesto que la verdad se define a través del impulso de la voluntad o de la invención, no existe un origen ni un fin al que toda experiencia, toda ley o saber, deban remitirse. Por consiguiente, no existen indicaciones que muestren a qué inclinarse y de qué alejarse. En todo caso, -y tal cual lo expresa una fragmento citado anteriormente- la lectura que deberá tener aquél que quiera leer la historia deberá ser política en el sentido de apuntar a ubicar las relaciones estratégicas de poder que conforman discursos de saber. Así como en el principio habíamos visto cómo Foucault plantea una reformulación para el estudio del poder, podría pensarse ahora que ese giro en la gramática del pensamiento también se plantea para la historia. La pregunta ya no se dirige a dar con su sentido, sino al análisis de su funcionamiento en tanto productora de efectos de verdad.

9 El título de esta segunda intempestiva lleva la marca de una polémica acerca de la "utilidad" que se propone; esta palabra no debe entenderse en términos pragmáticos y de racionalización técnica, sino más bien orientada a un tipo de conocimiento de la historia fecundo para la vida (Cano, 2003). 


\section{Referencias}

Bouton, C. (2005). Hegel et l'antinomie de l'histoire. Lecturas de Hegel. Ouvrage collectif sous la direction d'Olivier Tinland. Paris: Le livre de poche.

Cano, G. (2003). Nietzsche y la poderosa fuerza del presente. Una introducción a «Sobre la utilidad y perjuicio de la historia para la vida $»$. Sobre la utilidad y perjuicio de la historia para la vida. Madrid: Biblioteca Nueva.

Deleuze, G. (2008). Nietzsche y la filosofía. Barcelona: Anagrama.

Foucault, M. (2012). Lecciones sobre la voluntad de saber. Buenos Aires: FCE.

Foucault, M. (2006). Defender la sociedad. Buenos Aires: FCE.

Foucault, M. (2004). Nietzsche, la genealogía, la historia. Valencia: Pre-textos.

Foucault, M. (1995a). Nietzsche, Freud, Marx. Buenos Aires: Ediciones El Cielo por Asalto.

Foucault, M. (1995b). La verdad y las formas jurídicas. Barcelona: Gedisa.

Grüner, E. (1995). Foucault: una política de la interpretación. Nietzsche, Freud, Marx, Buenos Aires: Ediciones El Cielo por Asalto.

Nietzsche, F. (2003 [1874]). Sobre la utilidad y perjuicio de la historia para la vida. Madrid: Biblioteca Nueva.

Nietzsche, F. (2001 [1882]). La ciencia jovial. Madrid: Biblioteca Nueva.

Nietzsche, F. (1990). Sobre verdad y mentira en sentido extramoral. Madrid: Tecnos.

Nietzsche, F. (1984 [1885]). Más allá del bien y del mal. Buenos Aires: Hyspamerica.

Schleiermacher, F. (1996 [1829]). Ermeneutica. Milán: Rusconi. 